\title{
Modifying ways to perform ICU tracheostomies in COVID-19 patients: Approach to a safe and secure method
}

Kranti Bhavana ( $\sim$ bhavana.kranti@gmail.com )

All India Institute of Medical Sciences, Patna https://orcid.org/0000-0002-3417-1591

Arun Srinivaasan

All India Institute of Medical Sciences, Patna

Sheelia Ouseph

All India Institute of Medical Sciences, Patna

Swati Suneha

All India Institute of Medical Sciences, Patna

Rudra Prakash

All India Institute of Medical Sciences, Patna

Bhartendu Bharti

All India Institute of Medical Sciences, Patna

\section{Short Report}

Keywords: COVID 19, Bedside tracheostomy, Apnoeic tracheostomy, Aerosol generating procedure, carinal intubation

Posted Date: November 13th, 2020

DOl: https://doi.org/10.21203/rs.3.rs-100445/v1

License: (9) This work is licensed under a Creative Commons Attribution 4.0 International License.

Read Full License

Version of Record: A version of this preprint was published at Indian Journal of Otolaryngology and Head \& Neck Surgery on November 9th, 2020. See the published version at https://doi.org/10.1007/s12070020-02258-1. 


\section{Abstract}

Background-This study outlines the unique modifications to surgical tracheostomy procedure to combat the extraordinary situation the world has found itself in due to COVID 19 pandemic. We explain the modifications employed to the operative setup, anesthetic considerations and surgical procedure to enable us to provide timely and safe tracheostomy to the COVID ICU patients requiring it, while simultaneously maximally protecting our surgical personnel from the deadly exposure.

Methods- We conducted 55 surgical tracheostomies in severely sick ICU patients with the modifications deemed fit to achieve safe procedure for both the patient and the operating team. We analyzed the hospital record data of these patients and the surgical teams COVID 19 status to assesss the efficacy of our procedural modifications.

Discussion- The COVID 19 pandemic has thrown the entire medical fraternity into a dilemma as to how to provide the best possible care to the patients while protecting ourselves from its grip. Severely sick COVID patients often require tracheostomy for improved prognosis. We performed bedside open surgical tracheostomy and induced transient apnoea periprocedur along with carinal intubation. By making these simple and cost effective modifications to the procedure, we have ensured that patients get tracheostomised as and when required but not at the cost of the health and lives of our health care workers.

\section{Introduction}

COVID 19 is a novel pandemic viral infection by SARS COV-2, a positive sense single stranded RNA virus affecting millions worldwide without a definitive treatment. Although the disease is asymptomatic to mild in $80 \%$ of the affected people, around 17 to $35 \%$ of affected population progress to moderate to severe infection requiring care in High Dependency Units / Intensive Care Units with Non Invasive Ventilation(NIV)/High Flow Nasal Cannula. ${ }^{1,2}$ There is an unprecedented variable pattern of deterioration among such patients, requiring advanced airway support with ventilation.

Of all the hospitalised COVID 19 patients diagnosed with severe infection, approximately $60-70 \%$ have been found to develop ARDS requiring mechanical ventilation. Approximately $15 \%$ of these need invasive mechanical ventilation and eventually tracheostomy. ${ }^{3}$

Tracheostomy can be performed either by percutaneous route or by open surgical approach. Of late, there have been multiple guidelines with updates regarding the choice and practices of tracheostomy in COVID 19 patients. For patients with prolonged intubation, tracheostomy helps in improved tracheobronchial toileting, decreases dead space ventilation and reduces sedation requirement. Intubation related lung injury is reduced. It has also been found to result in better ventilation in many patients. All of this translates to better patient management and subsequent better prognosis. 
Our institute being the tertiary care centre for management of COVID illness has witnessed a rising trend of COVID 19 cases where patients requiring prolonged mechanical ventilation have also been on the rise. With an 80 bedded COVID ICU which has been fully occupied since May 2020, around 55 tracheostomises have been performed till September 2020.

We followed the international guidelines for open surgical tracheostomy for ensuring safety of the health care professionals involved in the procedure. Along with these precautions we also did a few procedural modifications to ensure it to be carried out smoothly in the ICU setup. These modifications would also protect the operating team of surgeons from undue exposure. In this article we would be discussing these modifications to ensure a safer tracheostomy procedure in an ICU setup during the COVID pandemic.

\section{Materials And Methods}

A total of 55 cases of open surgical tracheostomies were performed till September 2020 in the COVID ICU of All India Institute of Medical Sciences (AlIMS), Patna. Open surgical tracheostomy was preferred over percutaneous tracheostomy in view of -

1. The chances of de-recruitment and atelectrauma-associated pulmonary edema due to transient loss of PEEP while performing tracheostomy in ARDS patients is high. ${ }^{4}$ The total duration of transient loss of PEEP during tracheotomy and tube insertion was less in open surgical tracheostomy compared to percutaneous tracheostomy (PCT).

2. Open surgical tracheostomy is a cost-effective procedure as it does not involve using special equipment requirement of percutaneous tracheostomy.

3. Majority of the severe COVID 19 patients on prolonged ventilation have been found to have a deranged coagulation profile to hepatic involvement. An open surgical approach provides better surgical field visualisation, hence minimizing peri-operative blood loss.

As per the WHO guidelines, a designated COVID- 19 Operating Room (OR) would require a separate ventilation circuit from the OR COMPLEX with Bio Safety Level (BSL) 3 or above norms with negative pressure suction unit, isolated green corridors for patient transit and Anaesthetic bay. However, there are practical difficulties while shifting an intubated COVID patient to OR due to increased risk of environmental contamination as well as associated increased risk of viral exposure to the shifting team. This would require more manpower in terms of personnel handling shifting, receiving and sanitization. This eventually translates into increased Personal Protective Equipment (PPE) requirement which is always a matter of concern in view of the pandemic situation. Apart from this, to provide a single OR from a pre-established Modular Operating Theatre (OT) complex with isolated room ventilation and negative pressure suction room would be cumbersome in the least. These factors directed our preference towards performing bedside tracheostomy in all these COVID patients requiring the procedure.

Performing bedside tracheostomy, however came with its own set of challenges. ICU beds were broad with restricted height adjustments. There was a lack of dedicated overhead operating light source on 
each bed. This overall had a detrimental effect not only in terms of patient positioning but also increased direct exposure to the aerosols generated during the procedure due to bad spinal angle of the operating surgeon leading to aggravated bending towards the patient. With full gear PPE, visualisation is already compromised due to increased fogging. This combined with an uncomfortable surgeon's posture and inadequate lighting further compounded the problem.

All these above mentioned issues were overcome by a simple modification - Bedside tracheostomy on an OT table (Figure 1). We devised the idea of shifting a portable operating table, and portable operating light to the patient's bedside. This had the following advantages:

\section{a. Reduced Viral Exposure-}

Shifting an operating table by the bedside rather than shifting the patient to the operating room had a major advantage of exposing minimal number of health care workers to viral laden aerosols. Shifting the patient from their ICU bed to an adjacent bed requires minimal man power and less aerosol exposure due to less physical distance covered for shifting the patient. Shifting these intubated patients to OR involves disconnecting patient off from the main ventilatory circuit and shifting them on the Bain circuit where ventilation is performed manually by the anaesthetists. This is a potentially hazardous situation where aerosols are expelled at a much greater speed and can infect people all around. Thus an operating table by the side of ICU bed was one of the most feasible thing to do at that time.

\section{b. Better surgical environment-}

Availability of a portable OT light with sterile handles which can be manoeuvred by the surgeon, provides improved focussed surgical field lighting. This is crucial in situations such as a deep seated trachea which may be due to restricted neck extension in cases of cervical injuries, obese patients, and patients with a deviated trachea. Fashioning a bedside portable OT table excludes the need of COVID-19 OR changes that would otherwise have to be implemented. The ability to adjust height and position of the OT table also improves field visualisation dramatically.

\section{c. Better posture of operating surgeons -}

Adjustable height and surgical position functions of these portable OT tables provide better spinal angle and posturing for the operating surgeon and hence adds to her/his comfort. They can avoid unnatural bending towards the patient which in turn prevents direct aerosol exposure on to their faces. The need for precise surgical steps as well as timely surgical decision is affected by surgeon's fatigue status, which can be controlled by providing an optimal, posture to the operating surgeons. This is fulfilled better by a bedside operating room setup.

\section{d. Better appreciation of anatomical structures-}

Improved lighting of the surgical field provides better visualisation and appreciation of anatomical structures despite hampered visibility during PPE use. This can affect the final outcome in terms of 
surgical precision, operating time, intraoperative bleeding and perioperative complications. All this eventually contributes to decreased exposure time for the operating surgeon.

\section{e. Better patient outcomes-}

The incidence of atelectrauma and other transport associated complications are less owing to not requiring the patient to be disconnected from the ventilator for tracheotomy. This also aids in a better surgical outcome.

\section{f. A resource efficient \& cost effective alternative-}

Performing a bedside tracheostomy avoids need for a major OR modification, reduces the need for manpower, and subsequent PPE resource usage. Open surgical tracheostomy is cost effective in comparison to percutaneous tracheostomy as it does not require single-use PCT instrumentation set costing about INR Rs 7000-10,000.

Along with above surgical environment modifications, certain changes needed to be incorporated in the surgical steps of open tracheostomy. Most of the steps were in accordance with international guidelines given by various international bodies of Otolaryngology, Anaesthesia and Critical care. Few steps were improvised/added to protect the surgeons from undue aerosol exposure.

\section{Modified surgical Steps for COVID tracheostomy:}

1. Procedure under general anaesthesia- All patients were taken up for tracheostomy under complete general anaesthesia (GA). This aided us in achieving a good neck extension and respiratory control of the patient.

2. Avoiding utilisation of cautery - Electrocautery vaporises tissue particles and it can be a potent source of aerosol generation. Hence in place of it, ligatures and topical vasoconstrictors were used.

3. Injection of intra tracheal local anaesthetic drug- $0.5 \mathrm{ml}$ of intra tracheal $2 \%$ xylocaine was administered post confirmation via bubble test. This decreased the postoperative cough reflex.

4. Endotracheal tube clamping-Clamping of endotracheal tube (ET) at end inspiration/ inspiratory hold was done to avoid sudden de-recruitment and atelectrauma when tracheotomy was done.

5. Transient apnoea- We induced transient apnoea during tracheotomy to reduce aerosol generation, and subsequently the viral load to which the operating surgeon is exposed. Prior to making tracheal window, ET tube was progressed further till the carina and the cuff was maximally inflated. After tracheotomy and tracheal window creation, patient was put under transient apnoea and clamped ET tube was retrieved allowing the insertion of tracheostomy tube. Following this the tracheostomy tube cuff was inflated immediately and patient was re-ventilated. This helped us in keeping the duration of de-recruitment to a minimal. These changes were done to reduce the generation and exposure of surgeon to aerosols, who would otherwise be continually exposed. 
6. Use of inner cannula \& closed suction unit- Disposable inner cannula was routinely placed in all patients for better toileting. This also helped prevent the need for early tube change procedures due to tube obstruction. An in-line closed suction unit was used to suction tracheostomy tube to decrease environmental exposure.

7. Suturing the tracheostomy tube to the skin -Stay sutures for tracheostomy tube were placed in the flanks to prevent accidental decannulation and de-recruitment episodes during postural changes for prone ventilation.

8. Applying surgical mask to the stoma site A triple layered surgical mask was placed in between tracheostomy tube and ventilator circuit there by containing droplet contamination during stoma site dressing and from tracheostomy site leak (Figure 2).

\section{Discussion}

Since the beginning of COVID 19 pandemic, various guidelines and suggestions have come up for high aerosol generating procedures (AGP) like tracheostomy. Exposure and subsequent acquiring of COVID 19 disease among health care workers (HCW) has been attributed to COVID patient care. It has been a constant struggle to strike a balance between providing optimal patient care and ensuring the safety of HCWs. This has led to revisit and modify hospital environment and surgical steps to protect HCWs while performing AGPs.

The MERS pandemic provided the first clues and directions to deal with the current one. Those previous guidelines formed the basis of performing high AGPs during the COVID 19 pandemic. With time and understanding of the current situation, new recommendations were proposed based on current COVID 19 experience. Tracheostomy being deemed a high AGP, the focus has been on preventing aerosol spread during steps contributing the most to aerosol generation. Apneic tracheostomy with its variations, performing tracheostomy under the coverage of negative pressure drape and the choice of open surgical tracheotomy over percutaneous route have been considered towards achieving this goal. Percutaneous tracheostomy has been considered to generate aerosols at a higher rate than open procedure due to the need for bronchoscopy and multiple tracheal exchanges. ${ }^{5}$ Hence we preferred open surgical tracheostomy at our institute.

Prabhakaran et al. utilized negative pressure drapes for tracheostomy. Negative pressure hoods over the surgical field were theoretically expected to achieve a lesser degree of aerosol transmission. ${ }^{6}$ However, the actual amount of negative pressure required and the efficacy of the procedure remains unclear. Due to the sheer volume of patients requiring tracheostomy at our setup combined with the need for optimal utilization of already scarce resources, we decided against using them in our surgeries.

We went ahead with apnoiec tracheostomy for our patients. Variations in apneic tracheostomy included performing it under GA with complete muscle relaxant cover, proximal ET tube withdrawal followed by apnea induction prior to tracheotomy with or without progressing ET tube distally to reventilate, ${ }^{7,8}$ or 
progressing the ET tube upto the carina before tracheotomy. Though each variation has its expected benefits, none is strongly recommended above the other.

We encountered certain practical difficulties with apnea prior to tracheotomy. Most of these patients had severe ARDS and they critically desaturated, requiring a faster tracheostomy. There was a risk of accidental ET cuff damage during bubble test and/or tracheotomy, again leading to desaturation, loss of PEEP and de-recruitment.

The time taken to create a tracheal window was also higher in patients with ossified tracheal cartilage. Inducing apnoea post tracheotomy with carinal intubation circumvented these issues and provided us with the time margin needed to perform the procedure in a calm and collected manner thus avoiding potential complications, while simultaneously keeping the patient safe.

We found that none of our surgeons and the supporting technical team members were infected with COVID 19 during the entire duration we performed tracheostomies. This in itself has been a marker for efficacy of our modified surgical approach and our success in preventing infection among our team members. As the entire world is resorting to unprecedented measures to ensure both patient welfare and surgeon's safety, we propose the above modifications as a safe cost-effective way in achieving the same while performing tracheostomies.

\section{Conclusion}

Timely and safe tracheostomy is the need of the hour in the current pandemic scenario. Performing tracheostomy with technical modifications aiming at minimizing patient mobility and reducing aerosol generation has led us develop a safe procedure ensuring that our surgical team remains protected while performing it. Bedside apnoeic tracheostomy has also proven to be cost effective, rendering it efficient for both small and large hospitals alike.

\section{Declarations}

Compliance with ethical standards: All procedures contributing to this study have been conducted complying with the Helsinki Declaration of 1975 institutional guidelines. The INSTITUTE ETHICAL COMMITTEE AIIMS PATNA approved this study

Financial Disclosure: None of the authors have any financial disclosure to make

\section{References}

1. Docherty AB, Harrison EM, Green CA, et al. Features of 20133 UK patients in hospital with covid-19 using the ISARIC WHO Clinical Characterisation Protocol: prospective observational cohort study. BMJ. 2020;369:m1985. Published 2020 May 22. doi:10.1136/bmj.m1985. 
2. Grasselli G, Zangrillo A, Zanella A, et al. Baseline Characteristics and Outcomes of 1591 Patients Infected With SARS-CoV-2 Admitted to ICUs of the Lombardy Region, Italy. JAMA. 2020;323(16):1574-1581. doi:10.1001/jama.2020.5394.

3. Yang $X, Y u$ Y, Xu J, et al. Clinical course and outcomes of critically ill patients with SARS-CoV-2 pneumonia in Wuhan, China: a single-centered, retrospective, observational study [published correction appears in Lancet Respir Med. 2020 Apr;8(4):e26]. Lancet Respir Med. 2020;8(5):475-481. doi:10.1016/S2213-2600(20)30079-5.

4. Ghadiali S, Huang Y. Role of airway recruitment and derecruitment in lung injury. Crit Rev Biomed Eng. 2011;39(4):297-317. doi:10.1615/critrevbiomedeng.v39.i4.40.

5. Foster P, Cheung T, Craft P, Baran K, Kryskow M, Knowles R et al. Novel approach to reduce transmission of COVID-19 during tracheostomy. J Am Coll Surg. 2020. pii: S1072-7515 (20)303227. doi: 10.1016/j.jamcollsurg.2020.04.014.

6. Prabhakaran K, Malcom R, Choi J, et al. Open tracheostomy for COVID-19-positive patients: A method to minimize aerosolization and reduce risk of exposure. J Trauma Acute Care Surg. 2020;89(2):265-271. doi:10.1097/TA.0000000000002780.

7. Heyd CP, Desiato VM, Nguyen SA, et al. Tracheostomy protocols during COVID-19 pandemic. Head Neck. 2020;42(6):1297-1302. doi:10.1002/hed.26192.

8. Broderick D, Kyzas P, Sanders K, Sawyerr A, Katre C, Vassiliou L. Surgical tracheostomies in Covid-19 patients: important considerations and the "5Ts" of safety. Br J Oral Maxillofac Surg. 2020;58(5):585589. doi:10.1016/j.bjoms.2020.04.008.

\section{Figures}




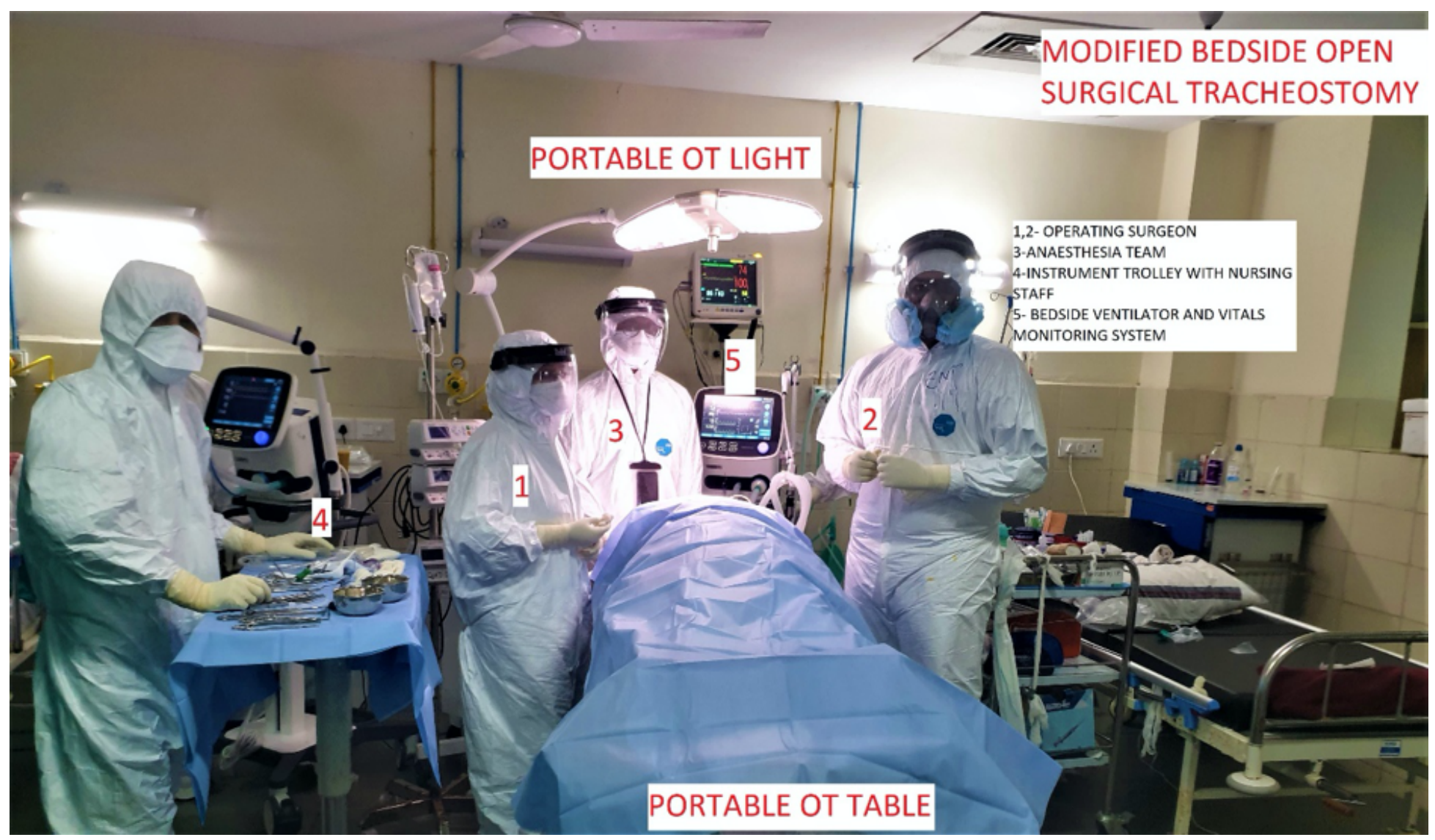

Figure 1

A movable OT table is shifted by the side of the ICU bed. Patient is shifted from his bed to the table without disconnecting the ventilatory circuit. 


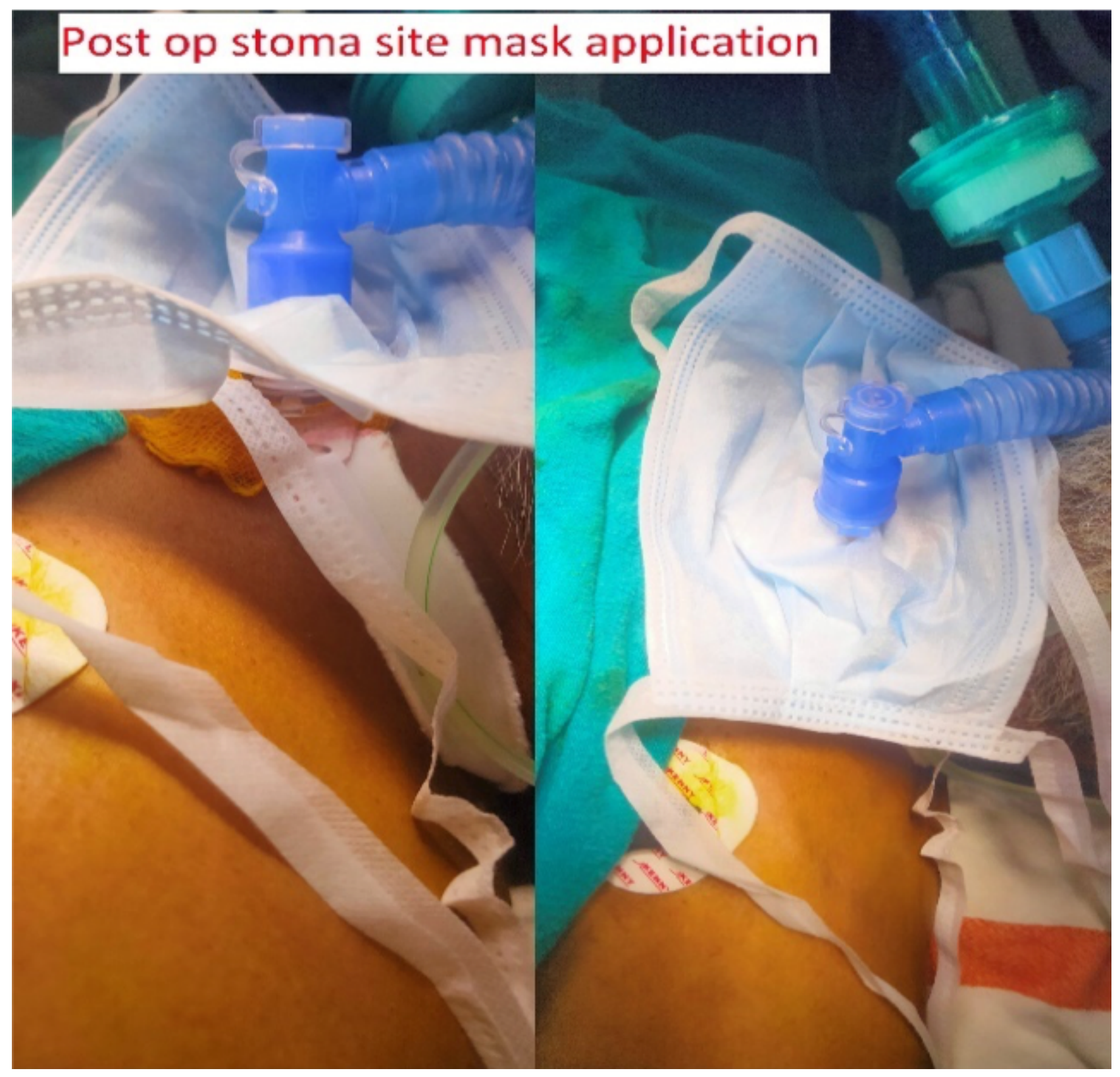

Figure 2

A surgical mask is applied in between tracheostomy tube and ventilator circuit there by containing droplet contamination 